\title{
A pregnant patient with fetal distress with severe post burn contracture of anterior trunk and perineum: A surgeon's nightmare!
}

\author{
Madhubari Vathulya, Meenakshi Joshi ${ }^{1}$ \\ Department of Plastic Surgery, Coronation Hospital, 'Department of Obstetrics and Gynecology, Doon Female Hospital, \\ Dehradun, Uttarakhand, India
}

Address for correspondence: Dr. Madhubari Vathulya, D-7/5, Street No 3, Vasant Vihar enclave, P.O New Forest, Dehradun - 248 006, Uttarakhand, India. Email: madhubari@yahoo.co.in

\section{ABSTRACT}

Introduction: A case report of a pregnant lady in the third trimester, presenting in the emergency with absent fetal movements is being described. A multi-speciality expertise was involved and a full term female baby was delivered. Presentation: The patient presented in a Government hospital, Dehradun, India. At the time of presentation the fetal heart sounds were absent and subsequently the severe post-burn deformity involving the anterior trunk, perineum and thighs were discovered. Management: Immediately a multi-speciality approach involving the plastic, obstetrics and pediatrics field were called for and an emergency caesarean with simultaneous contracture release with split skin grafts were performed after optimizing the patient for surgery. Conclusion: This is one of the first case reports reporting this rare combination of post burn contracture of trunk and perineum with fetal distress requiring emergency caesarean section along with release of abdominal and perineal post-burn contracture. This case interestingly reinforces that in such complicated cases a systematic and a combined multi-speciality approach still holds the key to patient's treatment even in district hospitals.

\section{KEY WORDS}

Post-burn contracture; pregnancy; abdominal burns; caesarean and burns

\section{INTRODUCTION}

pregnant lady with severe childhood post burn contracture of abdomen and perineum presented with fetal distress. Literature search revealed very few articles involving management of post-burn

\begin{tabular}{|l|l|}
\hline \multicolumn{2}{|c|}{ Access this article online } \\
\hline Quick Response Code: & Website: \\
\hline & www.jps.org \\
\cline { 2 - 2 } & DOI: \\
\hline
\end{tabular}

contracture with pregnancy. Articles related to post burn contracture neck release simultaneously during an elective caesarean and use of expanded abdominal apron after delivery to resurface the post burn contracture of upper abdomen have been described but there have so far been no articles that match our patient description.

\section{CASE REPORT}

\section{Presentation}

A 22 year old pregnant lady in the third trimester came to the emergency with absent fetal heart sounds in the third trimester in a government general hospital. Absent fetal heart sounds, meconium stained liquor and non 
progressive labour were the basis for considering the patient for emergency caesarean. The prognosis of the patient and the baby were explained clearly to the patient attendants. The patient in addition had severe post burn scarring due to burns in childhood and it extended from the chest region to the abdomen and perineal region with supra-clitoral hooding deformity. The left breast nipple-areolar complex was partially buried. Realizing the severity of contracture of the lower abdomen and perineum with distorted anatomy, the umbilicus lying very close to the mons region, the obstetrician realized the possible need for a plastic surgeon to reconstruct the huge defect once an incision for the caesarean section would be given which would not have been easy to close primarily.

\section{Operation details}

A combined multi-speciality expertise was involved including a plastic surgeon, an obstetrician and a pediatricianand an anaesthetiest. The patient was given spinal anaesthesia after optimizing her for surgery. An inverted $\mathrm{t}$ - shaped skin incision was planned in the lower abdomen region horizontally extending as far as the contracture and vertically extending as low as possible just stopping short of the clitoris when normal tissue was encountered and then the incision was converted to an inverted y-shape along both the sides of the remnant labias [Figure 1]. The rest of the caesarean section was completed in the usual way and a full born female baby weighing $2.75 \mathrm{~kg}$ was delivered. The baby was shifted to NICU for further observation. The anterior abdominal wall was closed in the usual way after delivering the placenta and the repaing the uterus. The mons pad of fat was found displaced and was recontoured to give the mound its normal shape. The skin defect extending from the lower abdomen to the upper thighs and

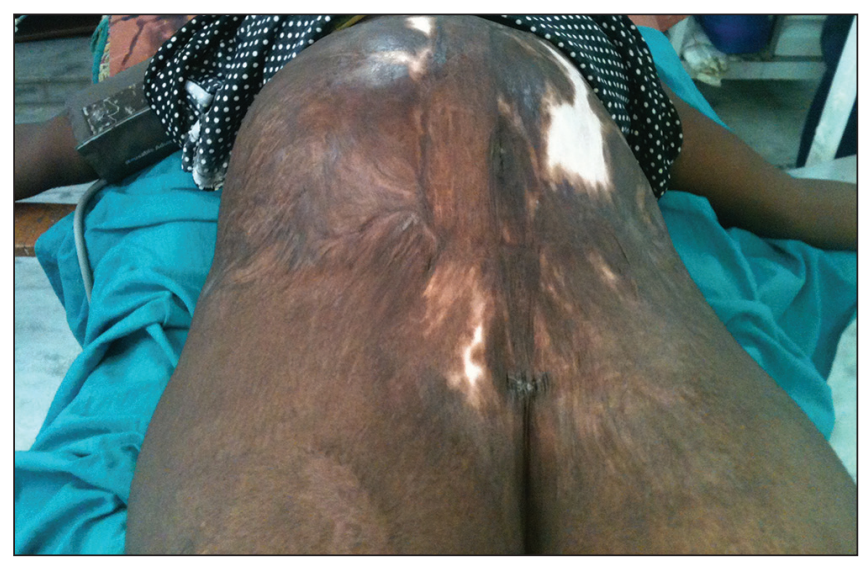

Figure 1: Post Burn scarring anterior abdominal wall - pre operative view anterior perineum [Figure 2] was resurfaced with skin grafts harvested from the thighs [Figure 3 and 4]. After infiltration of local anaesthetic, the left side nipple was everted along with the release of the gland tissue with skin graft cover as far as possible. But owing to the chronicity of the burns, the left breast had not attained the larche and hence she could lactate from the right breast only which seemed sufficient for the baby. She did not develop any breast engorgement on the left side however. The total operative time was 4 hours from the time of incision which included 20 minutes for the delivery of the baby. She received 3 units of packed red blood cells and 4 units of fresh frozen plasma in the perioperative period. Custom made splintage that extended from the abdomen to the thighs was provided for her for maintaining the thighs in the extended and abducted position. The baby was eventually handed over to the mother after sufficient monitoring.

\section{RESULT}

The graft take was satisfactory in spite of the initial maceration near the perineal areas. The patient can now walk with a near normal posture and has regained height postoperatively as compared to her previous stance as she used to walk in a crouched manner due to the severe post-burn contracture between the lower part of her body and the thighs. The patient was able to breastfeed her baby with her baby gaining weight in a normal manner.

\section{DISCUSSION}

Literature search has revealed lot of studies related to the management of the "acute burns" in pregnant

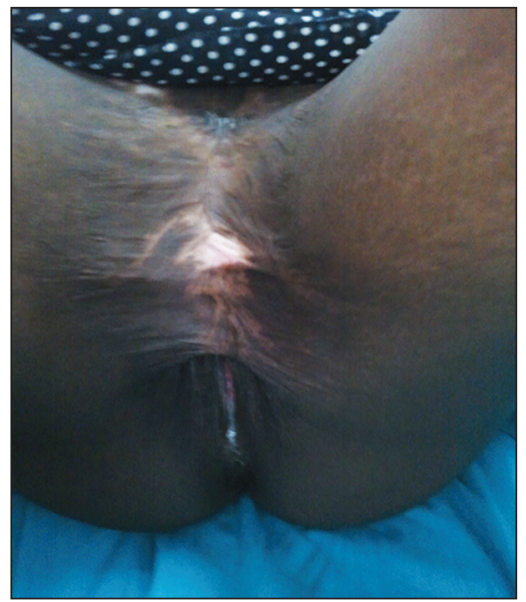

Figure 2: Perineal view of the Post burn contracture 


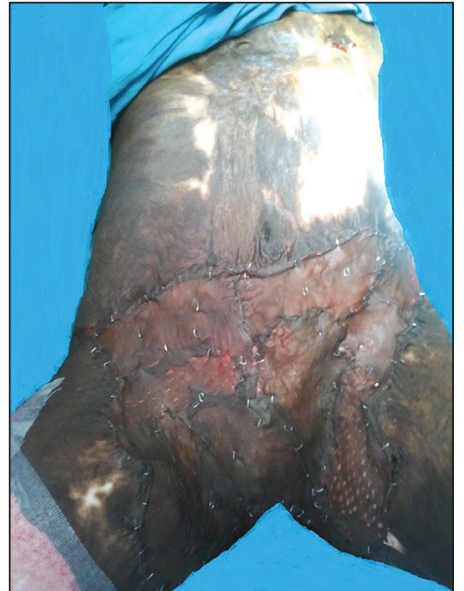

Figure 3: The postoperative view of the case with grafts in situ

patients with or without the requirement of caesarean section. This is probably one of the few case reports of a patient with such severe post burn deformity of the perineum and the lower abdomen to the degree that the clitoris, and the labias anterior $2 / 3$ almost invisible, presenting in the third trimester of her pregnancy due to childhood burns. Post burn contracture of the perineum is a rare burn sequel and the present case report is a secondary perineal burn contracture ${ }^{[1]}$ with burns not involving the perineum directly but causing obliteration of the perineum by forming scar bands across the external genitalia. The mean age of patients at time of burns in such cases is 7.6 years. ${ }^{[2]}$ In this case report the patient had sustained burns at the age of 6 years. The basic problems in such patients would be difficulty in sitting, standing, walking, squatting (common in Indians), urination ,defecation and sexual intercourse. These patients especially in the Indian setup are very embarrassed to reveal their difficulties and present so late as in our case in which the condition was revealed only during the plan of caesarean section. The effect of pregnancy on scars have been studied in detail by many authors and most predominantly have concluded that there is a higher incidence of keloids and hypertrophic scarring in pregnancy and the tendency decreases after menopause. ${ }^{[3-6]}$ It has been long assumed that pregnancy induced changes in the connective tissue of the abdominal wall are quite normal even in the severe burn scarring ${ }^{[7]}$ but this may not be always so especially in dense and circumferential abdominal burns. ${ }^{[8]}$ Very few surgical interventions for pregnant patients with severe burns are quoted in literature. In the initial trimesters of pregnancy the uterus is not so distended to be compromised by the pressure exerted by the tight abdominal wall due to scarring and hence these patients

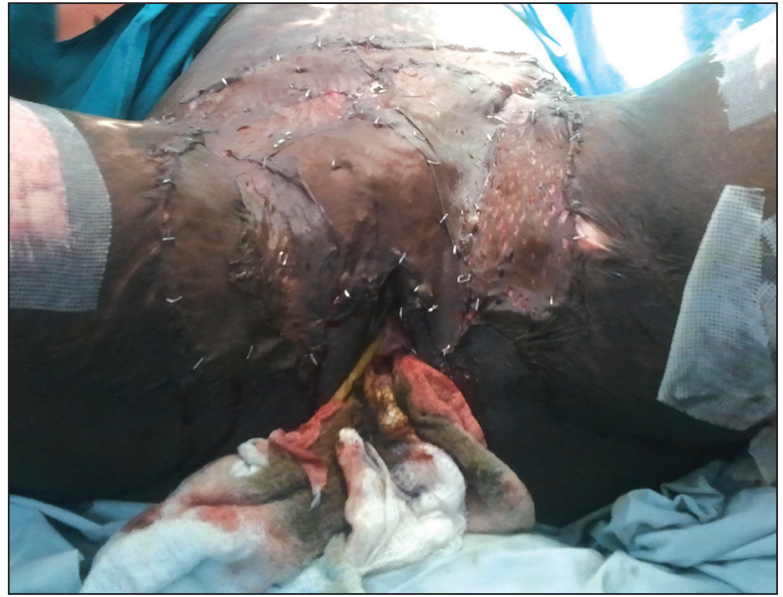

Figure 4: Postoperative photograph of the perineal view

predominantly present in the third trimester. There is no evidence to suggest increased risk of abortion even in severely scarred abdominal wall due to burns. ${ }^{[7]}$ Also surgical intervention till the completion of $2^{\text {nd }}$ trimester is generally avoided to prevent complications in the organogenesis of the foetus. In one study the indication for release of the contracture just after 24 weeks of gestation was the increasing pain perceived by the patient due to the tightness of the contracture band. ${ }^{[9]}$ In some of these cases after resurfacing with the skin graft during an ongoing pregnancy the patients have progressed successively to third trimester with normal vaginal delivery. ${ }^{[10]}$ If the scarring is to one side of the abdomen distortion may give a lopsided almost pendulous appearance. Mal-presentation is theoretically possible as with pendulous abdomen. ${ }^{[8]}$ In our case the absence of fetal heart sounds along with the meconium stained liquor and non progress of the labour were the indications of emergency caesarean. The patient's discomfort during the time of pregnancy was not that severe or probably the patient did not complain about it and she had not visited any hospital for a pre-natal checkup anytime before the actual time of delivery. Once the casesarean section incision was given by the obstetrician, a very large wound measuring nearly $15 \mathrm{~cm}$ in length was created in the lower abdomen. Hence the decision to involve the reconstructive surgeon was found appropriate for immediate reconstruction in the same stage. The majority of the contracture was released with the initial caesarean incision and hence the decision for completing the release was considered as the operating time would not have varied much. This provided the patient once in a life opportunity to return to normal life as her her low socioeconomic status would have prevented her from returning to 
the theatre for a staged reconstructive procedure. Also in these patients the lack of hygiene is a major concern and a large wound in the lower abdomen if left for a stage II reconstruction could get infected very easily. Even if they are kept in the hospital until the next surgery, keeping a critical newborn who had just recuperated from fetal distress close to a mother with a large wound area is not advisable for the chances of baby getting infected is logically high. Previous case reports which combined both procedures address pregnant patients with only lower abdominal burn scars subjected to abdominal wall reconstruction with the expanded uninvolved non-burnt upper abdominal flap. ${ }^{[1]}$ But in our case the upper abdominal wall had not expanded much due to probably the extensive nature of burns involving the chest and the whole of abdomen and anterior part of the perineum. A combination of elective caesarean with post burn contracture neck has also been reported ${ }^{[12]}$ in literature. Use of bilateral groin flaps ${ }^{[13]}$ or flaps of any other type of pedicled flap might not have solved the problem as the region of harvest was also a part of the scarred area in the patient and the defect to reconstruct was too large for groin flaps to cover. Moreover the patient was undergoing an emergency caesarean which requires short operative time with maximum benefit and the best option in such cases would be a split thickness skin graft resurfacing andhence the same was used. The graft shrinkage, hypertrophic scarring and keloid can be tackled with silicon gel sheets, pressure garments and massage but then the patient has to be alive and in the right frame of mind to follow these advices.

\section{REFERENCES}

1. Thakur JS, Chauhan CG, Diwana VK, Chauhan DC, Thakur A. Perineal burn contractures: An experience in tertiary hospital of a Himalayan State. Indian J Plast Surg 2008;41:190-4.

2. Kitzmiller WJ, Neale HW, Warden GD, Smith D. The effect of full-thickness abdominal wall burns sustained during childhood on subsequent childbearing ability. Ann Plast Surg 1998;40: 111-3.

3. Jain S, Jain M, Vaibhav J, Jain P. Hypertrophic scar and pregnancy. Healthcare 2013;1:15-8.

4. Moustafa MF, Abdel-Fattah MA, Abdel-Fattah DC. Presumptive evidence of the effect of pregnancy estrogens on keloid growth. Case report. Plast Reconstr Surg 1975;56:450-3.

5. Ford LC, King DF, Lagasse LD, Newcomer V. Increased androgen binding in keloids: A preliminary communication. J Dermatol Surg Oncol 1983;9:545-7.

6. Schierle HP, Scholz D, Lemperle G. Elevated levels of testosterone receptors in keloid tissue: An experimental investigation. Plast Reconstr Surg 1997;100:390-5.

7. Rai YS, Jackson DM. Childbearing in relation to the scarred abdominal wall from burns. Burns 1975;1:167-71.

8. Daw E, Mohandas I. Pregnancy in patients after severe abdominal burns. Br J Obstet Gynaecol 1983;90:69-72.

9. Haeskeer B, Green MF. A complication in pregnancy due to severe burns in childhood. Br J Plast Surg 1981;34:69-72.

10. Widgerow $A D$, Ford TD, Botha M. Burn contracture preventing uterine expansion. Ann Plast Surg 1991;27:269-71.

11. Takeda K, Sowa Y, Numajiri T, Nishino K. Expansion abdominoplasty for a pregnant woman with severe abdominal wall contracture due to a major burn during childhood: A case report. Ann Plast Surg 2013;70:643-6.

12. Kumari K, Ahuja V, Gombar S. Elective cesarean section in a parturient with post burn neck contracture: An anesthetic challenge! J Obstet Anaesth Crit Care 2013;3:44-6.

13. Grishkevich VM. Postburn perineal obliteration: Elimination of perineal, inguinal, and perianal contractures with the groin flap. J Burn Care Res 2010;31:786-90.

How to cite this article: Vathulya M, Joshi M. A pregnant patient with fetal distress with severe post burn contracture of anterior trunk and perineum: A surgeon's nightmare!. Indian J Plast Surg 2014;47:456-9.

Source of Support: Nil, Conflict of Interest: None declared. 